\title{
Le passage cénomanien-turonien dans les Monts des Ksour (Atlas Saharien Occidental, Algérie): biostratigraphie, géochimie et milieux de dépôt.
}

\section{Cenomanian-Turonian transition in Ksour Mountains (West Saharan Atlas, Algeria): Biostratigraphy, Geochemistry and deposit environments.}

\author{
Abdeldjebar Salhi ${ }^{1, *}$, François Atrops ${ }^{2}$, Miloud Benhamou $^{1}$ \\ ${ }^{1}$ Université Ahmed Ben Ahmed d'Oran II, Laboratoire de Géodynamique et Bilan des Bassins Sédimentaires, Département \\ de Géologie, Faculté des Sciences de la Terre et de l'Univers, 31000 Oran, Algérie. ORCID ID: https://orcid.org/0000-0002- \\ 3051-0066, https://orcid.org/0000-0003-4120-5099 \\ ${ }^{2}$ Université de Lyon 1, Laboratoire de Géologie, Bât. Géode, 2 rue Raphaël Dubois, 69622 Villeurbanne Cedex, France. \\ ORCID ID: https://orcid.org/0000-0003-3476-0684 \\ *Corresponding author: djebar2002@yahoo.fr
}

\section{RÉSUMÉ}

Dans les Monts des Ksour (Atlas saharien occidental), le passage cénomanien-turonien a été étudié sur le plan stratigraphique et géochimique à partir de deux coupes: Djebel M'daouer et Chebket Tamednaïa. Dans ces deux coupes, comme sur la plupart des plates-formes carbonatées peu profondes du domaine sud-téthysien, le faciès anoxique de la limite cénomanien-turonien matérialisé par des dépôts de "black-shales" est absent. En plus, la rareté des données biostratigraphiques rend difficile l'établissement d'une biozonation détaillée pour les deux coupes étudiées. Les deux seuls niveaux à rares ammonites de la Formation de Ghoundjaïa dans la coupe de M'daouer ont permis de caractériser les zones à Vibrayeanus, à Gamai et à Cauvini. Donc pour les deux coupes, il parait utile de recourir à l'étude isotopique qui a montré une zone d'excursion positive du $\delta^{13} \mathrm{C}$ s'étalant le long du Membre inférieur et la partie inférieure du Membre médian de la Formation de Ghoundjaïa. Cette zone d'anomalie est manifestée par trois pics marquants et caractéristiques du passage cénomanien-turonien et la limite entre ces deux étages se situe entre les pics 2 et 3 coïncidant avec le passage de la zone Gamai à la zone Cauvini. L'enregistrement de ces trois pics a rendu possible la corrélation locale de la coupe de Tamednaïa qui est dépourvue de faune valable sur le plan biostratigraphique.

Mots-clés: Limite cénomanienne-turonienne; Biostratigraphie; Géochimie; Atlas saharien; Ksour; Algérie.

\section{ABSTRACT}

In the Ksour Mountains (Western Saharan Atlas), the Cenomanian-Turonian transition has been studied stratigraphically and geochemically from two cross-sections: Djebel M'daouer and Chebket Tamednaïa. In these two sections, as on most of the shallow carbonate platforms of the South-Tethyan domain, the anoxic facies of the Cenomanian-Turonian boundary materialized by "black-shales" deposits are absent. In addition, the missing biostratigraphic data makes it difficult to establish a detailed biozonation for these two studied sections. The only

Recibido el 10 de julio de 2020; Aceptado el 5 de octubre de 2020; Publicado online el 26 de noviembre de 2020

Citation / Cómo citar este artículo: Salhi, A. et al. (2020). Le passage cénomanien-turonien dans les Monts des Ksour (Atlas Saharien Occidental, Algérie): biostratigraphie, géochimie et milieux de dépôt. Estudios Geológicos 76(2): e135. https://doi.org/10.3989/ egeol.44025.589

Copyright: (C) 2020 CSIC. This is an open-access article distributed under the terms of the Creative Commons Attribution-Non Commercial (by-nc) Spain 4.0 License. 
two levels with rare ammonites of the Ghoundjaïa Formation at the M'daouer section allow to characterize the Vibrayeanus, Gamai and Cauvini zones. So, for both sections, it seems useful to apply an isotopic study which showed a positive excursion zone of the $\delta{ }^{13} \mathrm{C}$ lying along the Lower Member and the lower part of the Middle Member Ghoundjaïa Formation. This anomaly zone is marked by three well-defined and characteristics peaks of the Cenomanian-Turonian transition and the boundary between these two stages stands between peaks 2 and 3 coincident with the transition from Gamai to Cauvini zones. The recording of these three peaks allowed the local correlation with the Tamednaïa section where fauna of biostratigraphic value is missing.

Keywords: Cenomanian-Turonian boundary; Biostratigraphy; Geochemistry; Saharan Atlas; Ksour; Algeria.

\section{Introduction}

Les anciens travaux sur les affleurements du Cénomanien et du Turonien dans les Monts des Ksour de 1'Atlas Saharien Occidental d'Algérie demeurent insuffisants et imprécis au point de vue biostratigraphique et sédimentologique. Les deux étages ont été reconnus pour la première fois par Flamand (1911), qui les a présentés sur sa carte géologique au $1 / 800.000^{\text {eme }}$, mais le détail de la succession restait imprécis. Il faut attendre les travaux de Cornet (1952), mais surtout de Bassoullet (1973), pour que soient décrites des coupes permettant de mieux connaître la succession des assises du Cénomanien-Turonien et que soient apportées de nouvelles données paléontologiques (notamment micropaléontologiques) et biostratigraphiques. A travers cette étude, dans leur ensemble les coupes des Monts des Ksour ne fournissent que peu d'éléments de datation. Les ammonites toujours rares, voire absentes, ne se rencontrent que dans quelques niveaux localisés et largement espacés, ce qui complique l'établissement d'une zonation détaillée et ne permet pas de situer facilement la limite CénomanienneTuronienne. Les deux seuls niveaux bien caractérisés et assez constants sont ceux des zones reconnus par Bassoullet. Ils ont été également mis en évidence dans plusieurs coupes (thèse en cours). Pour l'étude biostratigraphique et géochimique détaillées, le choix a été porté sur la coupe de M'daouer à série dilatée (Fig. 2) et ayant fourni le plus de données biostratigraphiques. La coupe de Tamednaïa se situe à environ $150 \mathrm{~km}$ vers le sud-ouest (Fig. 1), à série réduite et peu fossilifère a été étudiée à titre de comparaison. Il faut noter qu'aucune étude géochimique n'a été faite auparavant sur les niveaux de passage CénomanienTuronien des coupes de la partie occidentale des Monts des Ksour, à série dépourvue de dépôts de "black shales". La comparaison de la signature géochimique notamment les trois pics d'excursion positive du $\delta^{13} \mathrm{C}$ a permis d'établir des corrélations entre les niveaux du passage Cénomanien-Turonien des deux coupes étudiées. Aussi, la zone d'anomalie positive de la courbe $\delta^{13} \mathrm{C}$ a pu être repérée et le traçage de la limite cénomanienne-turonienne a été précisé. De même, cette étude nous a permet de tester l'efficacité du signal géochimique $\delta^{13} \mathrm{C}$ dans des séries de plates-formes peu profondes pas ou peu documentées sur le plan biostratigraphique.

\section{Méthode de traitement géochimique}

Les échantillons prélevés sur un pas d'un mètre environ dans les coupes du Djebel M'daouer et de Chebket Tamednaïa, ont été utilisés pour la réalisation des lames minces aux laboratoires du Centre de recherche et développement (CRD) de Boumerdès et au Laboratoire de Géologie à l'Université de Claude Bernard, Lyon I. L'étude du microfaciès a permis la caractérisation des textures et la fraction allochimique et orthochimique selon Dunham (1962) et Folk (1959). Une partie des échantillons a été broyée en poudre, pesé puis soumis à la réaction d' $\mathrm{HCl}(10 \%)$ sous l'appareil «Bernard » pour l'analyse de la calcimètrie. Après étalonnage, la lecture sur éprouvette graduée du volume de $\mathrm{CO}_{2}$ dégagé suite à la réaction d'acide est portée sur un tableau de données à partir desquelles, une courbe d'évolution du taux de $\mathrm{CaCO}_{3}$ est tracée le long de chaqu'une des deux coupes étudiées.

L'analyse des isotopes stables a été choisie sur des morceaux ou fragments de roches homogènes d'échantillons sélectionnés, intacts et non affectés par la fissuration ou la dolomitisation et ne contenant pas d'éléments allochémiques comme des bioclastes, par exemple. Pour cette analyse isotopique, les échantillons préparés sont concassés et broyés à l'aide d'un broyeur ordinaire où approximativement $5 \mathrm{~g}$ de la roche totale en poudre sont requis pour une analyse complète de la roche. Un broyeur à billes de céramique est utilisé pour la réduction en poudre des 


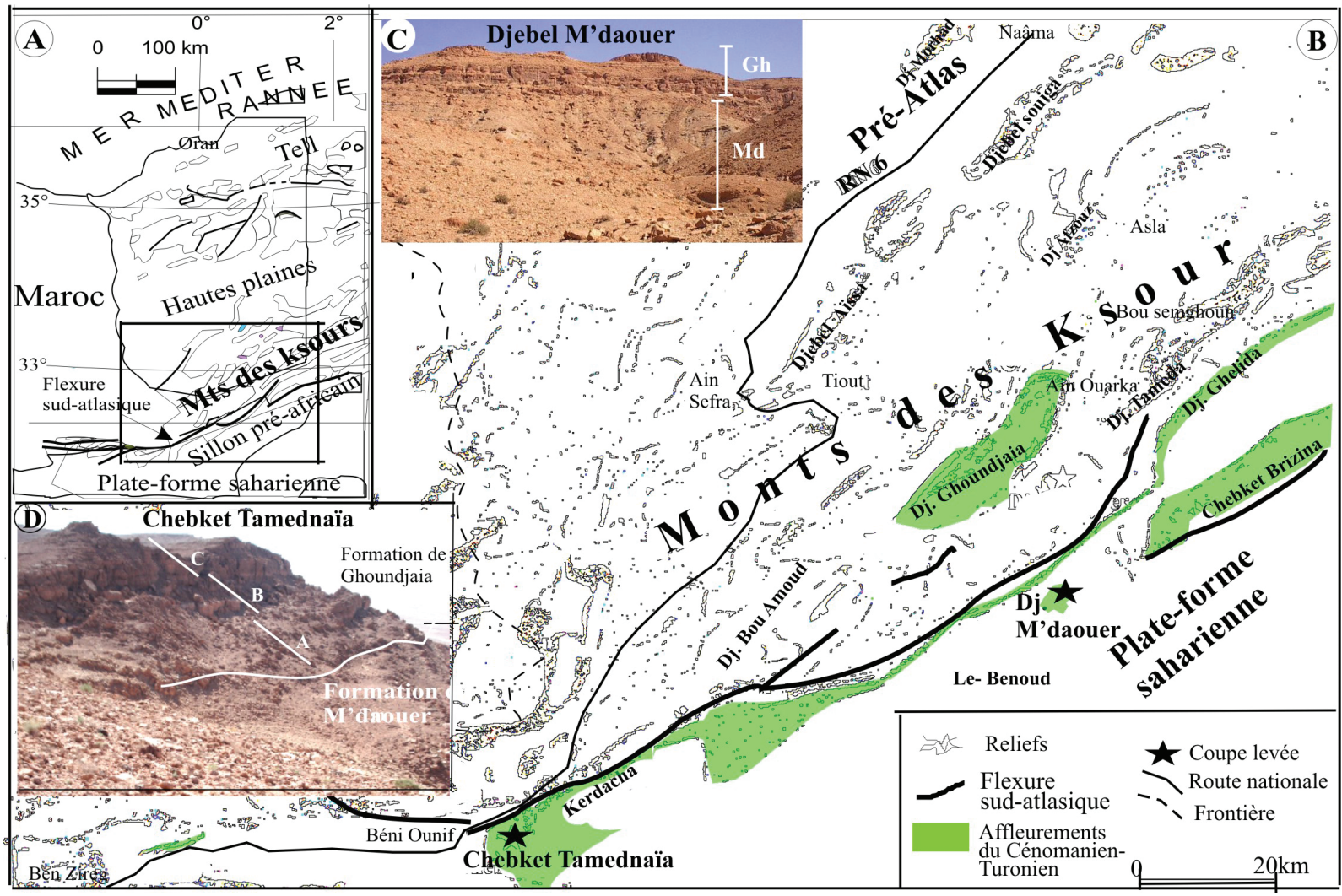

Fig. 1.-A. Carte du nord-ouest algérien et situation l'Atlas Saharien; B. Carte des Monts des Ksour montrant les affleurements du Cénomanien-Turonien et la localisation des coupes étudiées du Djebel M'daouer et Chebket Tamednaïa. Les coupes levées sont indiquées en étoiles. C-D. Vues panoramiques des coupes de M'daouer et Tamednaïa respectivement.

échantillons qui sera, ensuite, représentative et prête pour l'attaque à l'acide phosphorique sous une température de $25^{\circ} \mathrm{C}$. Le $\mathrm{CO}_{2}$ dégagé est séparé grâce à un jeu d'extraction impliquant de l'azote liquide $-270{ }^{\circ} \mathrm{C}$, puis de la neige carbonique $-50{ }^{\circ} \mathrm{C}$. Les poudres obtenues sont ensuite passées en fusion et dosage grâce au spectromètre de masse de l'Université Claude Bernard de Lyon. Les données des isotopes stables $\delta^{13} \mathrm{C}$ sont reportées en (\%o) relatifs par rapport au standard PDB (Pee Dee Belemnite, Crétacé des Etats-Unis). La mesure de ces standards se faisait au même moment que les échantillons permettent d'obtenir la précision analytique des mesures: $\delta^{13} \mathrm{C} \pm 0.03 \%$.

\section{Cadre géologique des Monts des Ksour}

Les coupes étudiées de Djebel M'daouer (Fig. 1C) et de Chebket Tamednaia (Fig. 1D), distantes de $150 \mathrm{~km}$, font partie du versant sud-ouest des Monts des Ksour, à proximité de la flexure sud-atlasique. La série sédimentaire du Cénomanien-Turonien est sub-tabulaire avec un pendage très faible vers le sudest, et formant une dalle ou barre calcaréo-dolomitique surmontant une vire marneuse (Fig. 1C-D). Au sud-est, les Monts des Ksour sont limités par le sillon pré-africain et la plate-forme saharienne, au nord-est par le Pré-Atlas et les Hauts-plateaux, à l'est par le Djebel Ben-Zireg et au nord-est par Chebket Brizina (Fig. 1 A-B). Les affleurements du CénomanienTuronien longent la flexure sud-atlasique et forment de larges synclinaux crétacés à fond plat encadrés par des anticlinaux jurassiques longs et étroits caractéristiques du style "jurassien" des Monts des Ksour. Cette flexure sépare ces monts de la plate-forme saharienne où les terrains crétacés sont peu affectés par la tectonique alpine (Busson et al., 1999) à l'exception de la phase compressive de l'Aptien. Durant l'Albien-Turonien un régime d'extension à grande envergure jalonne le nord-est de la chaine atlasique 


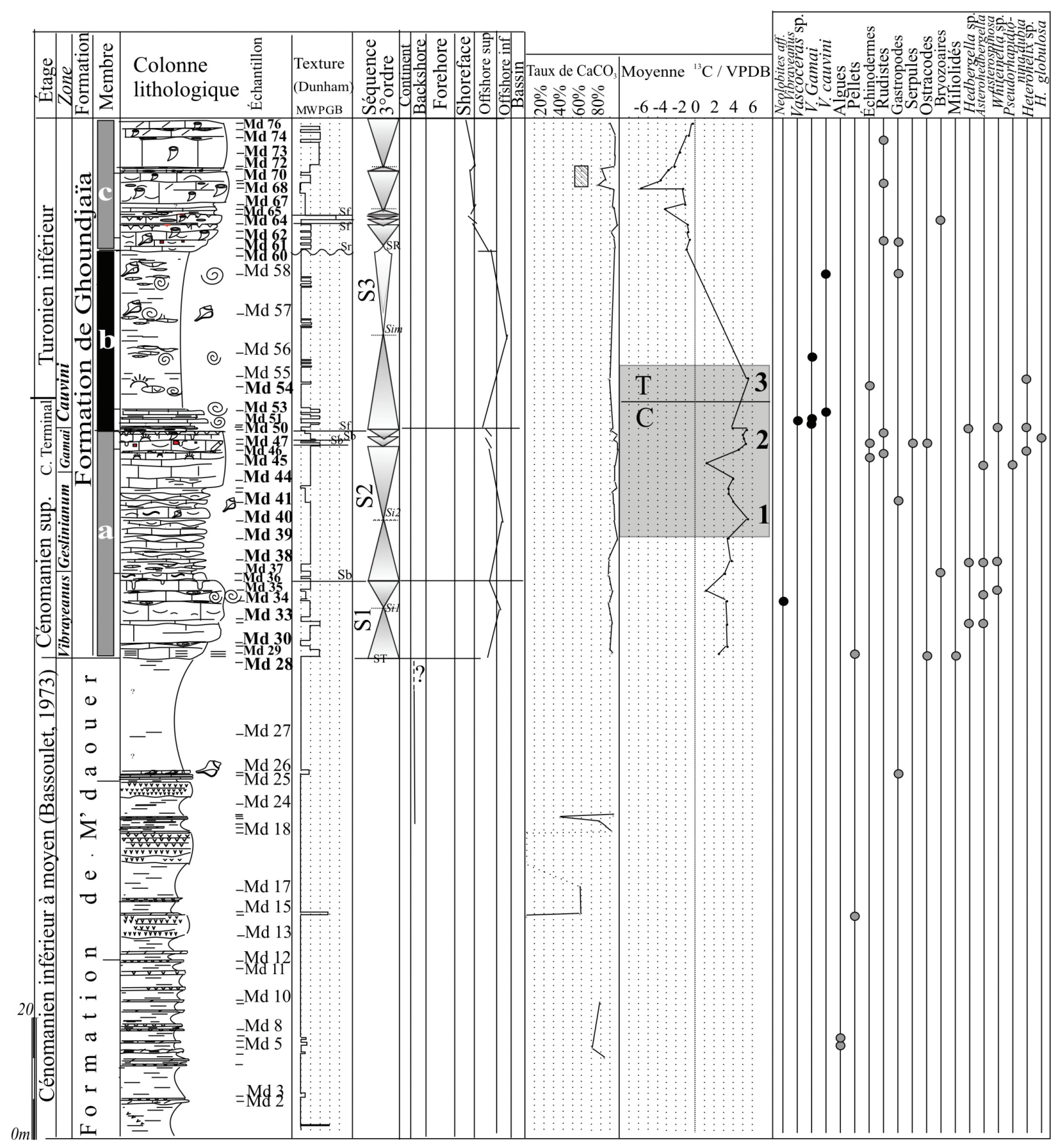

Fig. 2.- Succession lithologique, biozones d'ammonites, répartition de la microfaune, évolution séquentielle, courbes isotopiques du $\delta^{13} \mathrm{C}$ et calcimétrie, dans les niveaux de passage Cénomanien-Turonien du Djebel M'daouer. Voir légendes sur la figure 6.

créant ainsi un système de grabens et demi-grabens qui contrôle la distribution des carbonates de plate-forme et les faciès profonds du bassin, plus particulièrement les faciès riches en matière organique de l'intervalle cénomanien-turonien (Guiraud, 1990; Aris et al. 1998; Herkat et Delfaud, 2000;
Herkat, 2001). Les Mésétas limitrophes de ce système, correspondraient à des blocs stables peu ou non déformés par les évènements compressifs du Cénozoïque (Frizon de Lamotte et al., 2000). Le passage cénomanien-turonien est considéré comme un intervalle de changement de dynamique d'extension 
d'un synrift (subsidence mécanique) à un post-rift (subsidence thermique), associé à une composante latérale de transtension en "pull-appart" (Laville, 1985; Kazi-Tani, 1986).

\section{Stratigraphie et microfaciès}

La série crétacée étudiée des Djebels M'daouer et Chebket Tamednaïa comprend deux formations définies par Bassoulet (1973): M'daouer et Ghoundjaïa. A Djebel M'daouer, la Formation de M'daouer repose en concordance sur la Formation de Rhélida (Bassoullet, 1973). Dans la coupe de Tamednaïa, elle est discordante sur la Formation de Tiout qui fait partie de la succession désignée comme "Continental intercalaire" (Conrad, 1969). Seule la Formation de Ghoundjaïa à faciès plus marin fera l'objet d'une étude géochimique dans les deux coupes.

\section{Coupe du Djebel M'daouer}

Cette coupe de 170,50 m correspond au Djebel M'daouer; elle se subdivise en deux formations. La Formation de M'daouer d'âge Cénomanien inférieur à moyen (Bassoullet, 1973) superpose sans discontinuité la Formation de Rhélida d'âge Albien terminal (Bassoullet, 1973) qui est essentiellement formée de marnes et de dolomies. La limite inférieure de la Formation de M'daouer est liée à l'apparition des premiers bancs gypseux. La deuxième formation, également définie par Bassoullet, est nommée Formation de Ghoundjaïa d'une épaisseur de $92 \mathrm{~m}$ et forme une barre essentiellement carbonatée. Sa limite inférieure est un passage progressif entre les marnes et les calcaires sans discontinuité apparente.

\section{Formation de M'daouer}

Elle a été rapportée au Cénomanien inférieur par Bassoullet (1973) par comparaison avec une coupe similaire décrite par Cornet (1952) au nord d'ElBiodh. Cette formation épaisse de 78,50 m, se subdivise en trois membres (Fig. 2, 3A).

Membre inférieur (Md 2-Md 12; 32m), marneux à gypse et comprend des passées dolomicritiques à pelloïdes et gravelles (Md 3-Md 5; Fig. 4A) et à structures cryptalgaires irrégulières affectées par des bird-eyes et sheet-cracks (Fig. 4B).
Membre médian (Md 13-Md 24; 28 m). Comprenant trois masses métriques de gypse, il contient également quelques niveaux à pellets bien classés associés à des gravelles ( Md 15). Les cavités de la dolomicrite (Md 17; 0,10 m) à bird-eyes et sheetcracks sont remplies de cristaux de gypse fibreux, ponctuellement silicifiés (Fig. 4C).

Membre supérieur ( $\mathrm{Md} 25-\mathrm{Md} 28 ; 21 \mathrm{~m})$, marneux à gypse, il contient une lumachelle dolomitique à gastéropodes de taille centimétrique $(1-3 \mathrm{~cm})$ à tests recristallisés (Md 26, Fig. 4 D) et à remplissage géotrope tantôt micritique, tantôt gypseux.

Cette formation caractérise un dépôt lagunaire supratidal (backshore) proche de l'émersion.

\section{Formation de Ghoundjaïa}

Epaisse de $92 \mathrm{~m}$, cette formation essentiellement carbonatée $\left(90 \%\right.$ de $\left.\mathrm{CaCO}_{3}\right)$ est composée de trois membres (Fig. 2, 3A) :

Membre inférieur (Md 28-Md 50; $39 \mathrm{~m}$ ). Il est carbonaté et commence par une masse calcaire, mal stratifiée (Md 28 - Md 37) de texture wackestone-packstone dolomicrosparitique et laminée à ostracodes (Md 29; Fig. 4E) et miliolidés. Cette masse est succédée par un niveau (Md 30) de dolo-pelmicrosparite à bioclastes diffus, puis un niveau (Md 32-Md 34) de biomicrite mudstone-wackestone à foraminifères: Whiteinella sp. (Fig. 4F), Asterohedbergella asterospinosa (Hamaoui, 1965) (Fig. 4G), Hedbergella sp. et des ostracodes. Le niveau sus-jacent (Md 35) est une biodolomicrite bioturbée ayant livré dans le niveau Md 35 plusieurs spécimens d'ammonites Neolobites aff. vibrayeanus (d'Orbigny, 1841) (Fig. 3C). La partie médiane de ce membre (Md 38-Md 45), est formée d'une succession de bancs décimétriques de biomicrite bioturbée ondulée, à texture mudstone-wackestone et dont les interbancs de marnes feuilletées à ostracodes et foraminifères: Hedbergella sp. (Fig. 4 H, Md 38) et Asterohedbergella asterospinosa (Hamaoui, 1965), Pseudorhapydionina dubia (De Castro, 1965) (Fig. $5 \mathrm{~A}, \mathrm{Md} 45)$ associés à des bioclastes d'échinodermes, de bivalves, de gastéropodes (Md 41) et de bryozoaires. La partie supérieure du membre (Md 46-Md 50), est marquée par une lumachelle (Md 46) à bivalves et contenant de petits oursins, ostracodes et Heterohelix sp. Cette lumachelle est surmontée 

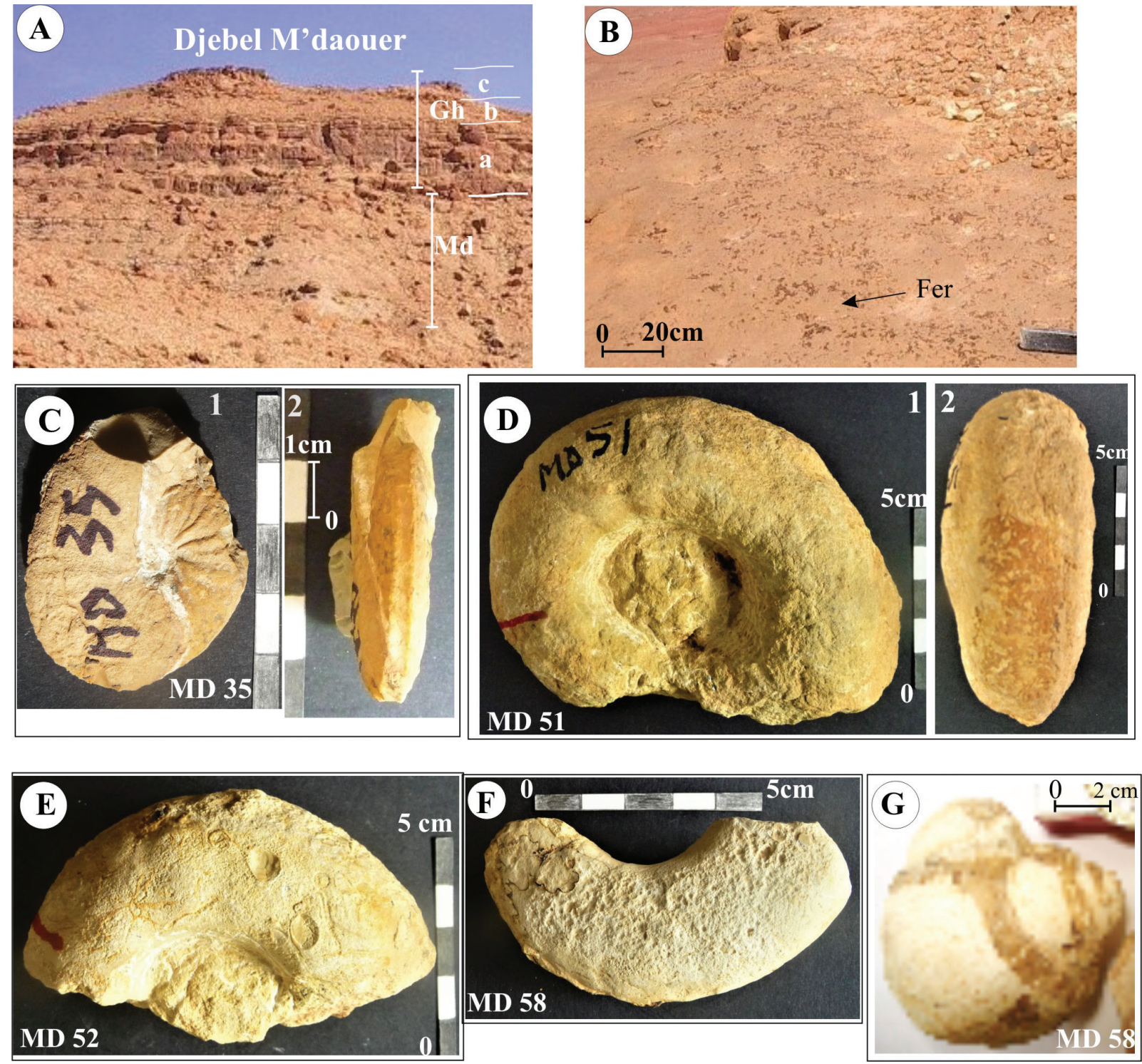

Fig. 3.- A. Photo du Djebel M'daouer montrant la Formation de M'daouer (Md) surmontée par la Formation de Ghoundjaia (Gh), avec ses trois membres (a, b et c). B. Surface durcie ferrugineuse au toit du Membre inférieur de la Formation de Ghoundjaia, au Djebel M'daouer. C, Exemplaire légèrement déformé de Neolobites aff. Vibrayeanus (d'Orbigny), Md 35, D= $41 \mathrm{~mm}$, vues latérale et ventrale, Formation de Ghoundjaia, Djebel M'daouer. D, Vascoceras gamai (Choffat), Md 51, D = $96 \mathrm{~mm}, \mathrm{O}=33 \mathrm{~mm}(\mathrm{O} / \mathrm{D}=0,34)$. A D = 82 $\mathrm{mm}, \mathrm{H}=33 \mathrm{~mm}, \mathrm{E}=35 \mathrm{~mm}(\mathrm{E} / \mathrm{H}=1,06)$. Noter la présence de gros tubercules périombilicaux émoussés sur la moitié postérieure de la loge. Le trait rouge marque le début de la loge. Formation de Ghoundjaia, Djebel M'daouer. E. Vascoceras gamai (Choffat), MD 52, Djebel M'daouer, $\mathrm{D}=78 \mathrm{~mm}, \mathrm{O}=25,5, \mathrm{O} / \mathrm{D}=0,32, \mathrm{H}=28,5, \mathrm{E}=32,5, \mathrm{E} / \mathrm{H}=1,14$. Présence de deux gros renflements périombilicaux émoussés sur le début de la loge. La loge montre des empreintes en creux d'épizoaires intratalames (Serpules) indiquant une longue flottaison de la coquille. Présence aussi de petites coquilles de bivalves fixées ultérieurement sur le moule interne induré. Le trait rouge marque le début de la loge. F. fragments de Vascoceras cf. Cauvini (Chudeau), Md 58, Formation de Ghoundjaia, Djebel M'daouer G. Gastéropode Tylostoma sp., Md 58, Formation de Ghoundjaia, Djebel M'daouer.

par deux bancs de biomicrite wackestone renfermant des mollusques bivalves, des gastéropodes, des échinodermes, des annélides (serpulidés) (Md 47, Fig. 5 B), des ostracodes et des foraminifères benthiques, surtout des Heterohelix globulosa (Ehrenberg, 1884)
(Md 48-Md49, Fig. 5 C) et Heterohelix sp. Le sommet de ce membre, niveau Md 50, est une biodolomicrite scellée par une surface durcie, bioturbée et ferrugineuse bien marquée, contenant des articles de crinoïdes et des Whiteinella sp. (Fig. 5 D). 

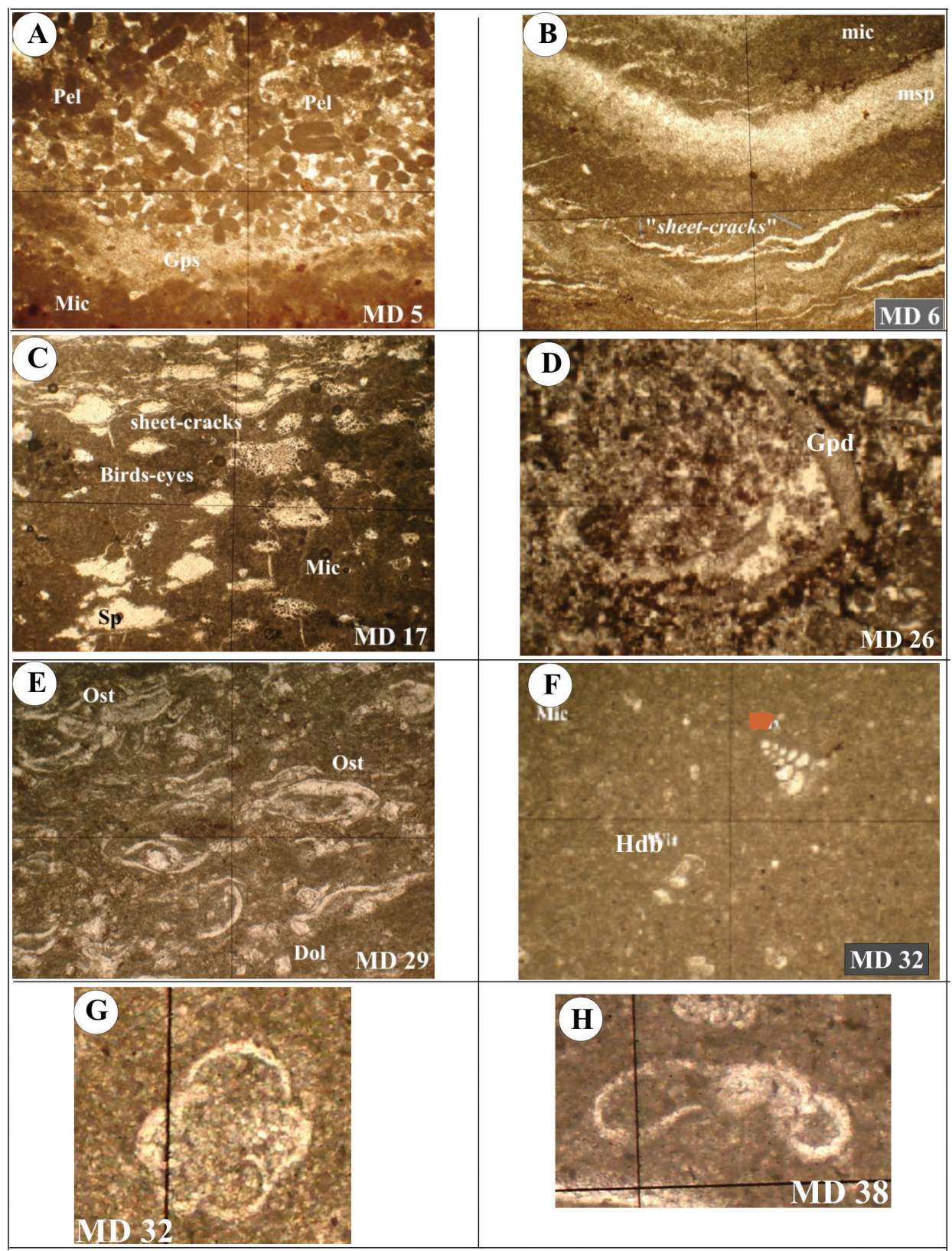

Fig. 4.- Microfaciès et microfaune de quelques niveaux de la coupe de M'daouer. A. Dolomicrite laminés à pellets (pel) et gravelles. La lamine micritique (mic) s'alterne avec une lamine pelmicrosparitique, intercalés par une plage gypseuse (gps) Md 5, Formation de M'daouer, Djebel M'daouer. X40. B. Lamination ondulée cryptoalgaire entre micrite (mic) et microsparite (msp) engendrant parfois des craquelures type "sheet-cracks Md 6, Formation de M'daouer, Djebel M'daouer. X10. C. Bird-eyes et sheet-cracks remplis de sparite (sp) dans une matrice micritique (mic) Md 17, Formation de M'daouer, Djebel M'daouer. X10. D. Dolomicrosparite à test de gastéropodes recristallisé (Gpd) Md 26, Formation de M'daouer, Djebel M'daouer. X10. E. Wackestone-packstone biodolomicrosparitique laminée à ostracodes (Ost) et rares miliolidés Md 29, Formation de Ghoundjaïa, Djebel M'daouer. X40. F, Mudstone-wackestone biomicritique à Whiteinella sp., Hedbergella sp. (Hdb) Md 32, Formation de Ghoundjaïa, Djebel M'daouer. X40. G. Mudstone-wackestone biomicritique à Asterohedbergella asterospinosa (Hamaoui, 1965), Formation de Ghoundjaïa, Djebel M'daouer, Md 32. X100. H. Mudstonewackestone biomicritique à Hedbergella sp. Md 38, Formation de Ghoundjaïa, Djebel M'daouer. X100. 


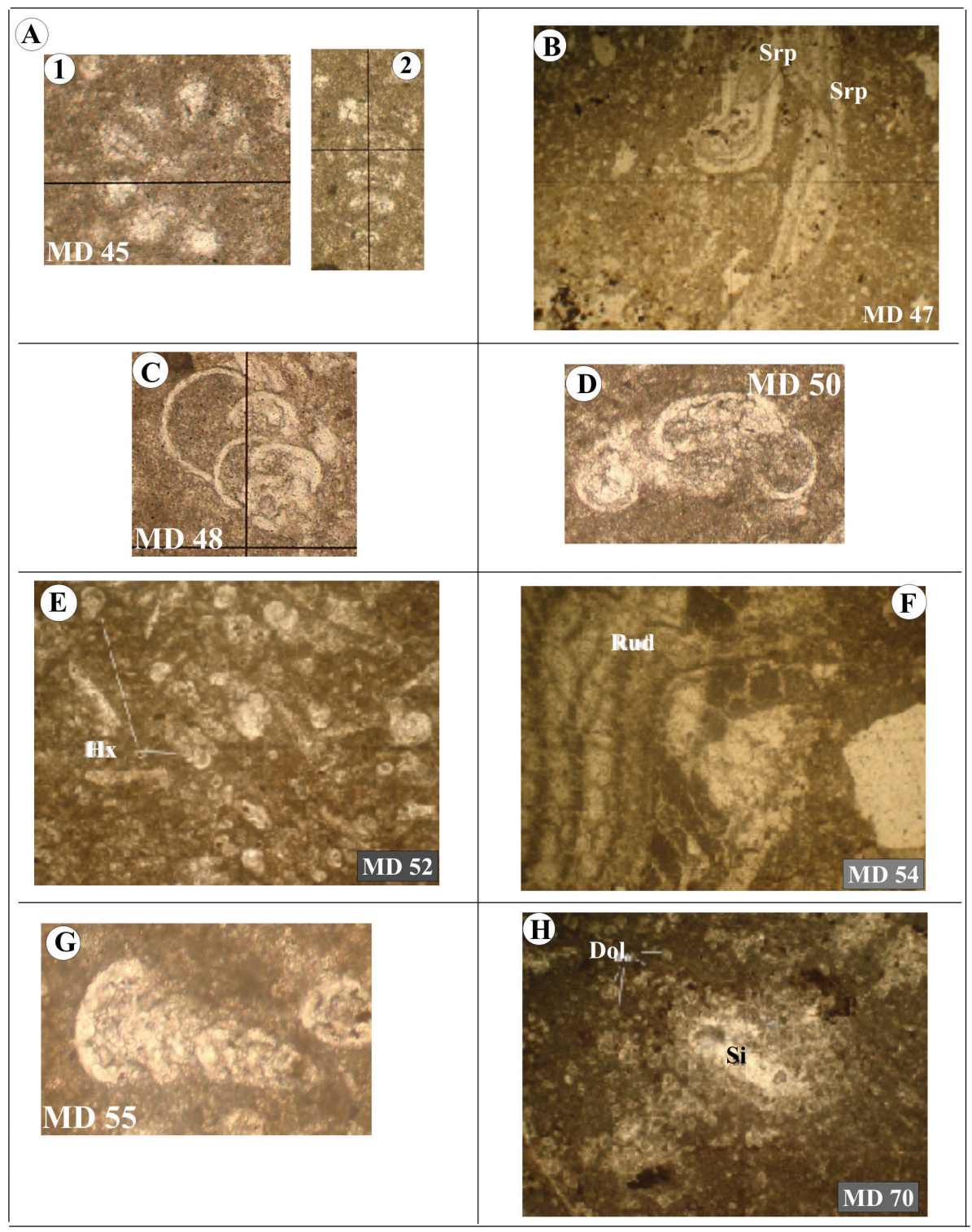

Fig. 5.- Micofaciès et microfaune de la coupe de M'daouer. A. Pseudorhapydionina Dubia (De Castro, 1965); (1) section transversale, (2) section longitudinale Md 45, Formation de Ghoundjaïa, Djebel M'daouer. X100. B. Biomicrite wackestone finement bioclastique et contenant des bioclastes indeterminables et des serpulidés (Srp) Md 47, Formation de Ghoundjaïa, Djebel M'daouer. X40. C. Heterohelix globulosa (Ehrenberg, 1884) Md 48, Formation de Ghoundjaïa, Djebel M'daouer. X100. D. Whiteinella sp. Md 50, Formation de Ghoundjaïa, Djebel M'daouer. X100. E. Dolomicrosparite argileuse grisâtre à Heterohelix sp. (Hx) abondants Md 52, Formation de Ghoundjaïa, Djebel M'daouer. X40. F. biomicrite argileuse jaunâtre friable à débris de rudistes (Rud) Md 54, Formation de Ghoundjaïa, Djebel M'daouer. X10. G. Textularia sp. Md 55, Formation de Ghoundjaïa, Djebel M'daouer. X100. H. Wackestone biodolomicritique bioturbé à silex (Si) et rhomboèdres de dolomite (Do) Md 70, Formation de Ghoundjaïa, Djebel M'daouer. X10.

Membre médian (Md 51-Md 60; $31 \mathrm{~m}$ ), essentiellement marneux, il débute par trois minces bancs (Md 51-Md 53), marno-calcaires ondulés à la base et à stratification irrégulière, noduleuse à pseudo-noduleuse. Deux bancs (Md 51, Md 52) de dolomicrosparite argileuse grisâtre ont livré des mollusques bivalves et de nombreuses ammonites dont Vascoceras gamai (Choffat, 1897) (Fig. 3 D-E), Vascoceras cauvini (Chudeau, 1909) et Vascoceras sp., ainsi que des gastéropodes, des 
échinodermes, des ostracodes et des foraminifères planctoniques du genre Heterohelix (Fig. 5E). Le reste de ce membre est formé de marnes vertes à rares passées de calcaires marneux noduleux ( $\mathrm{Md}$ 54-Md 60) qui montrent à leur base (Md 54), des plaquettes centimétriques de biomicrite argileuse, mudstone ayant livré des bivalves, y compris des débris de rudistes (Fig. 5F)., de rares ammonites mal conservées et des échinodermes. Le banc Md 55 est une biomicrite argileuse bioturbée et bioclastique à grumeaux et de mudstone-wackestone qui a livré quelque Vascoceras et des Textularia sp. (Fig. 5G). Ensuite, le niveau Md 56-Md 58 de marno-calcaires noduleux à terriers contient de rares Vascoceras cf. cauvini mal conservées (Fig. 3F) et des Tylostoma sp. (Fig. 3G).

Membre supérieur (Md 61-Md 76; 22 m), carbonaté, il est représenté par des calcaires partiellement dolomitisés contenant des silex et des rudistes. Il débute par des calcaires biomicritique bioturbés et noduleux de texture mudstone wackestone (Md 61-Md 62) contenant de rares gastéropodes, bivalves, oursins, foraminifères et bryozoaires, suivis de six minces bancs (Md 63-Md 66) marno-calcaire dolomitisés stratodécroissant à silex et rudistes montrant une biodolomicrite bioturbée de type wackestone. Au-dessus, une surface ferrugineuse, le banc (Md 67) à rudistes, est surmonté par un banc ( $\mathrm{Md}$ $68-\mathrm{Md} 70,1,50 \mathrm{~m}$ ) calcaire massif, formée de biodolomicrite bioturbé très bioclastique et montrant le microfaciès de type mudstone à wackestone. Ce banc contient des rudistes, bryozoaires, échinodermes, radioles d'oursins et bivalves, ainsi que de petits foraminifères planctoniques. Le banc ( $\mathrm{Md} 70)$ dolomitique et contenant des silex (Fig. 5H) est surmonté par un niveau (Md 71-Md 76) de calcaire mudstone à bryozoaires, mollusques bivalves et échinodermes.

La présence de foraminifères, des miliolidés et d'ostracodes à la base du Membre inférieur de la Formation de Ghoundjaïa, indiquent le début d'ouverture de la région étudiée sur le milieu marin, qui rapidement approfondit vers les conditions de l'offshore supérieur avec l'arrivée des ammonites Neolobites et des foraminifères planctoniques (fig. 2 , 9) correspondant ainsi une première inondation (Si1) de la séquence de dépôt "S1". La partie médiane de ce membre, correspond à une deuxième phase d'inondation (Si2 de la "S2") toujours dans les conditions de l'offshore supérieur qui est marquée par la disparition brutale de la faune et la microfaune (Fig. 2). Une troisième phase d'inondation plus prononcée (Sim) vers l'offshore inférieur a eu lieu au milieu du Membre médian de la séquence de dépôt "S3", précédée par un renouvellement faunique (base de "S3"). Cette phase est marquée par l'arrivée massive des groupes de Vascoceras Cauvini (Chudeau, 1909) et Vascoceras Gamai (Choffat, 1897) et par un nouveau épanouissement des foraminifères planctoniques: Whiteinella sp., Heterohelix sp., Hedbergella sp. et Pseudorhapydionina dubia (De Castro, 1965). Le Membre supérieur voit le retour à des conditions relativement moins profondes avec des cortèges saccadés de haut niveau marin relatif de la partie supérieure de la séquence "S3", matérialisé par le dépôt de biomicrites et/ou dolomicrites contenant des fragments et quelques patch-reefs disséminés de rudistes qui caractérisent les conditions de shoreface.

\section{Coupe de Chebket Tamednaïa}

Cette coupe (Fig. 1, 6) moins épaisse que la précédente $(61 \mathrm{~m})$, correspond à Chebket Tamednaïa, situé à environ $150 \mathrm{Km}$ au sud-ouest de la coupe précédente. Elle est composée par deux formations: La Formation de M'daouer et la Formation de Ghoundjaïa définies par Bassoullet (1973). La première superpose en discordance angulaire la partie supérieure de la Formation de Tiout (Bassoullet, 1973 ) essentiellement gréseuse. Dans cette coupe, la Formation de Rhélida est absente.

\section{Formation de M'daouer}

Elle est essentiellement marneuse et moins épaisse que la précédente (17 m) (fig. 6-7A)

Membre inférieur (TM 6-TM 11; 7,50 m), est formé de marnes verdâtres devenant blanchâtres à passées de calcaires silteux rouges pseudonoduleux se terminant par un banc de calcaire blanc $(0.10 \mathrm{~m})$ enduit par une surface ferrugineuse durcie (sf).

Membre supérieur (TM 12-TM 15; 9,50 m), est formé de marnes vertes $(6 \mathrm{~m})$ livrant dans leur partie supérieure (TM16) quelques rares huîtres et devenant jaunâtres, renfermant des passées onduleuses de calcaires pseudonoduleux. Ces calcaires ont une texture wackestone dolomicritique et sont riches en 


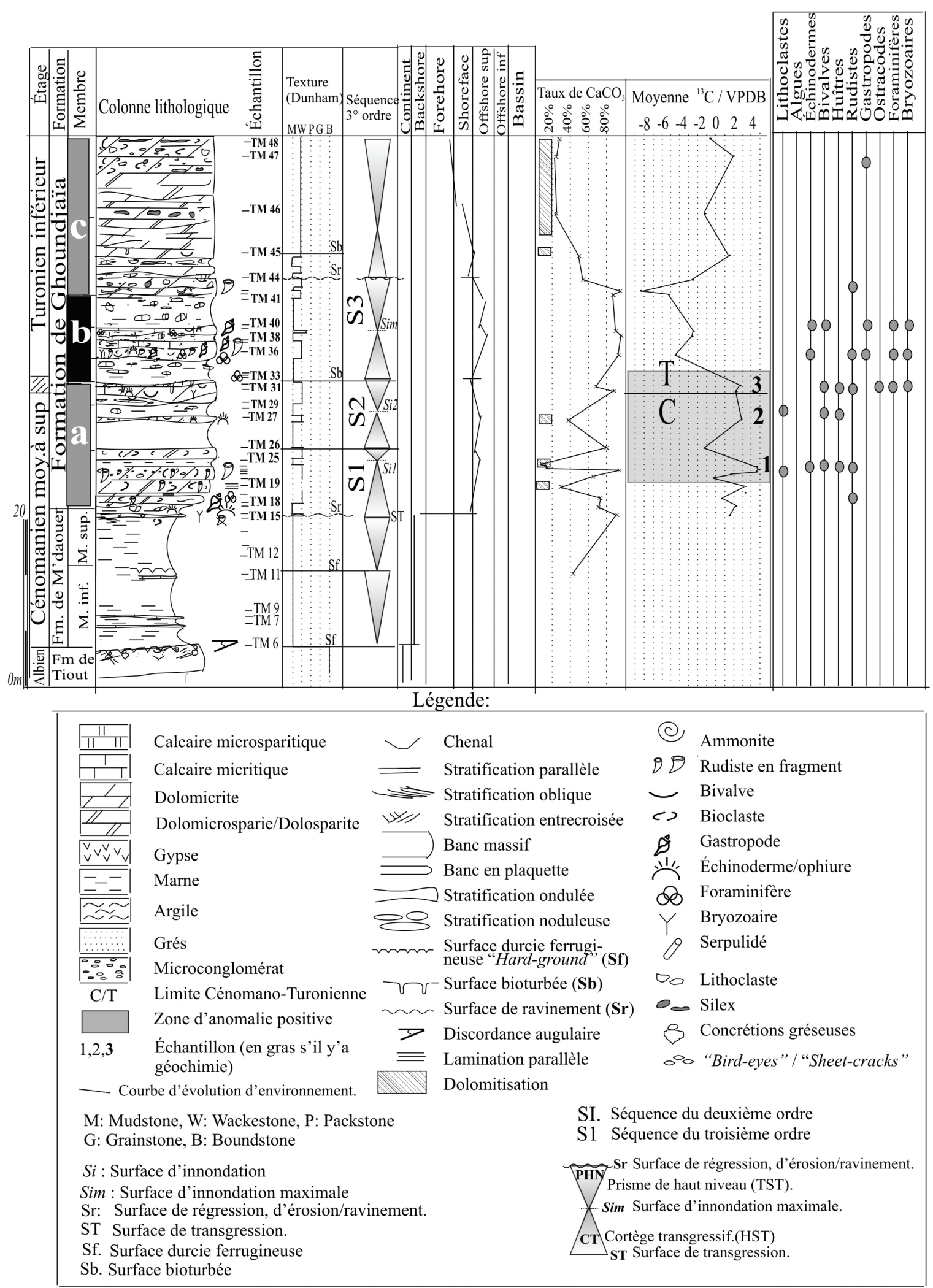

Fig. 6.- Succession lithologique, répartition de la microfaune, évolution séquentielle, courbes isotopiques du $\delta{ }^{13} \mathrm{C}$ et de la calcimétrie, dans les niveaux de passage Cénomanien-Turonien de la coupe de Chebket Tamednaïa. 

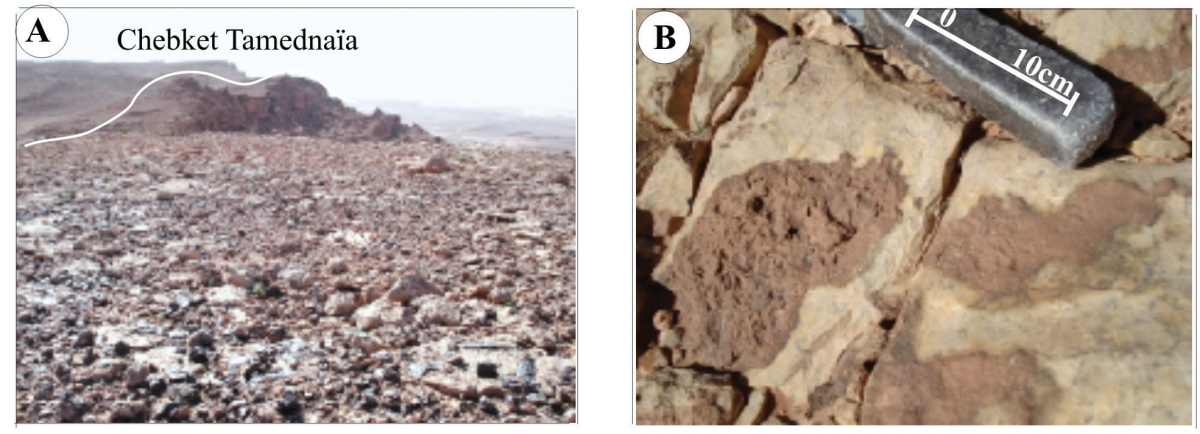

Fig. 7.- A. Vue sur Chebket Tamadnaïa montrant l'ondulation à l'échelle décamétrique au toit de la Formation de Ghoundjaia. B. Surface bioturbée avec remplissage par de la micrite rouge (TM 33) de la discontinuité au toit du Membre inférieur de la Formation de Ghoundjaia, de la coupe de Tamednaïa.

bioclastes de bryozoaires, rudistes et échinodermes et contenant de rares foraminifères planctoniques dans le niveau TM 15.

Le faciès marnes rouges plus ou moins silteuses et non fossilifère de la Formation de M'daouer de cette coupe indiquent des conditions lagunaires supratidales (backshore) et montrant de rares courtes invasions marines marquées au sommet du membre par la présence de foraminifères et de débris de bioclastes.

\section{Formation de Ghoundjaïa}

Epaisse de $44 \mathrm{~m}$, cette formation se subdivise en trois membres.

Membre inférieur (TM 18-TM 33;14 m), calcaréo-dolomitique, il débute par une dolomicrosparite à fantômes de rudistes (TM 18-TM 24; 7,50 m) passant à une dolomicrite laminée (TM 18), puis une intrabiomicrite de texture wackestone (TM 20, $0,20 \mathrm{~m}$ ) à rudistes. Ensuite, un biostrome de rudistes (TM 21) est terminé par une dolosparite silteuse.

Vers le sommet, un banc ondulé et ravinant (TM $31,2,50 \mathrm{~m})$ de dolomicrosparite contenant des nodules de biomicrite mudstone à bivalves, radioles, plaques d'échinodermes, bryozoaires et petits foraminifères. Ce membre se termine par un niveau (TM 33) de micrite finement bioclastique montrant des plages rougeâtres à éléments détritiques. Il s'agit d'une discontinuité bien marquée (Fig. 7B).

Membre médian (TM 34-TM 41;12,50 m). Il débute par des marnes blanches, passant progressivement à des calcaires noduleux et pseudo-noduleux à bivalves (TM 35). Viennent ensuite trois bancs de calcaire mudstone-wackestone biodolomicritique à bioclastes. Le niveau TM 36, contient: rudistes, échinodermes, bryozoaires, gastéropodes puis des calcaires wackestone-packstone riches dans le niveau TM 39 en foraminifères, grands bivalves, débris d'échinodermes et bryozoaires.

Membre supérieur (TM 42-TM 48; 19 m), dolomitique, débute par un banc de dolomicrosparite beige contenant dans le niveau TM 43 des rhomboèdres de dolomite à nucléi sombre et des fantômes de rudistes passant à une dolomicrosparite vacuolaire (TM 44), correspondant à des traces de dissolution de bioclastes; puis à un niveau dolosparitique ondulé et bioturbé (TM 45). Ce membre se termine par une dolomicrosparite blanche (TM 48) à silex en nodules devenant stratiformes vers le sommet.

Le faciès de la base du Membre inférieur de la Formation de Ghoundjaïa montre une ouverture du milieu moins prononcée vers l'Offshore supérieur (Si1) de "S1" marqué dans le niveau TM 20 par des intercalations de lithoclastes (Fig. 6). Sa partie supérieure marque une deuxième phase d'inondation (Si2) de "S2" terminée par un niveau (TM 31) bioturbé rougeâtre (Fig. 7B) à tendance à l'émersion. Au-dessus, la présence de rares Whiteinella évoquent une troisième phase d'inondation de l'offshore (Sim) de "S3" (Fig. 6). Le shoreface est atteint qu'à partir de la base du Membre supérieur très dolomitisé qui présente à son toit des ondulations décamétriques caractéristiques de la zone tidale (Fig. 7A). Ces structures sédimentaires sont équivalentes aux sand-waves décrites dans le Djebel Askar en Tunisie (Grosheny et al., 2013). 


\section{Cadre biostratigraphique de la coupe de Djebel M'daouer}

En référence aux données biostratigraphiques issues de travaux réalisées par Collignon (1957, 1965), Amard et al. (1981), Amedro et al. (1996) et Busson et al. (1999) sur les ammonites du plateau du Sahara algérien (Tinhert), le calage des niveaux paléontologiques de la région d'étude a pu être établie suivant la zonation d'ammonites établie par ces auteurs. Ces derniers reconnaissent dans l'intervalle du Cénomanien supérieur-Turonien inférieur quatre zones:

C1. Zone à Calycoceras guerangeri comprenant des Neolobites vibrayeanus (d'Orbigny, 1841) qui sont présents dans la coupe de M'daouer;

C2. Zone à Metoicoceras geslinianum qui correspond à l'intervalle qui n'a pas donné d'ammonites dans la coupe de M'daouer et il est situé entre le niveau à Neolobites vibrayeanus (D'Orbigny, 1841) et le niveau à Vascoceras gamai (Choffat, 1897).

C3. Zone à Neocardioceras judii. Dans la coupe de M'daouer, elle comprend les espèces Vascoceras gamai (Choffat, 1897) et Vascoceras cauvini (Chudeau, 1909).

C4. Amedro et al. (1996) considère que la limite cénomanienne-turonienne correspond à la disparition des Vascocera gamai (Choffat, 1897) espèce qui cependant disparait même avant la limite du Cénomanien-Turonien (Meister \& Piuz, 2013).

C5. Zone à Watinoceras coloradoense. Dans la coupe de M'daouer, l'espèce Vascoceras cf. cauvini (Chudeau, 1909) pourrait dater la base du Turonien.

La Formation de Ghoundjaia dans la coupe du Djebel M'daouer montre deux niveaux principaux à ammonites (Fig. 2). Le premier (Md 35), situé dans la partie inférieure du Membre inférieur, a livré de nombreux Neolobites vibrayeanus (D’Orbigny, 1841) (Fig. 3C) qui caractérisent la zone Vibrayeanus (Fig. 8). Le deuxième (Md 51-52), à l'extrême base du Membre médian, renferme de fréquents Vascoceras gamai (Choffat, 1897) (Fig. 3 D-E) qui indiquent la zone Gamai du sommet du Cénomanien supérieur. Au-dessus, dans le niveau Md 58, on rencontre de rares Vascoceras aff. cauvini (Chudeau, 1909) mal conservés (Fig. 3F).
Les ammonites sont toujours rares dans le Turonien inférieur des Monts des Ksour. Les spécimens dans le Membre médian de la Formation de Ghoundjaïa, se situent au-dessus du niveau riche en Vascoceras gamai, (Choffat, 1897) ce sont des Vascoceras fragmentaires, non déterminables. Un spécimen mieux conservé, provenant de la partie supérieure du Membre médian (banc Md 58, Fig. 2) est attribué à Vascoceras cf. cauvini (Chudeau, 1909) (Fig. 3 F). Cette espèce pourrait donc encore persister à la base du Turonien, en association avec des formes du Turonien inférieur.

Ces données paléontologiques permettent, d'une part, de caractériser clairement les zones à Vibrayeanus, à Gamai et à Cauvini du Cénomanien supérieur et d'autre part, de montrer que la discontinuité qui termine le Membre inférieur est située entre les zones à Gamai et à Cauvini (Fig. 2, 8), et qu'il n'y a pas de lacune biostratigraphiquement décelable à ce niveau. Enfin, la limite Cénomanien-Turonien se situe au sein du Membre médian, à un niveau qu'il est actuellement impossible de préciser par les ammonites.

L'intervalle d'épaisseur $25 \mathrm{~m}$, situé entre le niveau à Neolobites vibrayeanus (d'Orbigny, 1841), et le niveau à Vascocera gamai (Choffat, 1897) n'a pas livré d'ammonites (Fig. 2, 8), ce qui ne permet pas de lui assigner un âge précis. Il semble être corrélatif sans doute, pour l'essentiel, à la zone standard à Geslinianum, si on compare le profil isotopique du carbone $\left(\delta^{13} \mathrm{C}\right)$ de la coupe de $\mathrm{M}^{\prime}$ daouer et celui du stratotype du Turonien à Pueblo (Colorado, USA), qui est bien daté par les ammonites. Ces mêmes données géochimiques nous conduisent à placer la limite inférieure de la zone standard à Geslinianum au niveau du Membre inférieur, et le début du Turonien (base de la zone à Devonense), dans la partie inférieure du Membre médian (Fig. 2, 8).

\section{Données de la géochimie isotopique et corrélation}

Globalement, le niveau anoxique de la limite cénomanienne-turonienne "OAE-2 » (Oceanic Anoxic Event) définie par Schlanger \& Jenkyns (1976) correspond à un intervalle de stockage du carbone organique (Schlanger et al., 1987; Arthur et al., 1988; Jenkyns et al., 1994), sous la forme de "black shales", laminés 


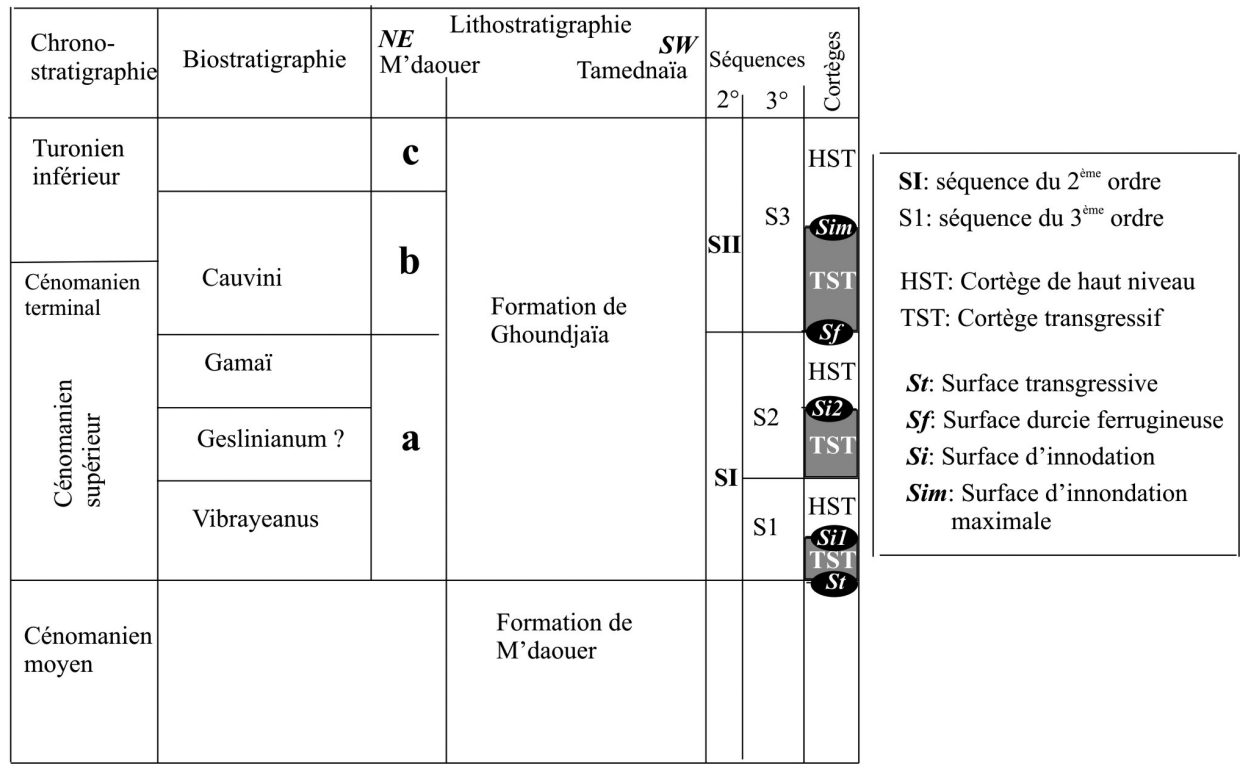

Fig. 8.- Tableau illustrant le cadre lithostratigraphique, biostratigraphique et séquentiel des niveaux de passage cénomanienturonienne de Djebel M'daouer et de Chebket Tamednaïa.

et riches en matière organique (sup. 1\% COT). En domaine téthysien, l'EAO-2 correspond à une augmentation du $\delta{ }^{13} \mathrm{C}$, observée sur les carbonates $(+$ $2,5 \%$ en moyenne) comme sur la matière organique ( $+4 \%$ en moyenne) (Accarie et al. 1996, Jarvis et al. 1988, Schlanger et al. 1987). Le calibrage biostratigraphique de la courbe représentative des variations isotopiques de $\delta{ }^{13} \mathrm{C}$ confère à cette anomalie positive un caractère synchrone susceptible d'être utilisé pour des corrélations à grandes distances (Accarie et al., 1996, Jarvis et al., 1988, Schlanger et al. 1987). On sait que la plupart des études géochimiques du $\delta^{13} \mathrm{C}$ se font sur les séries profondes "black shales" alors que peu de données géochimiques sont établies sur les séries de plate-forme peu profondes.

Le taux de $\mathrm{CaCO}_{3}$ (calcimètrie) dépasse les $90 \%$ dans presque la totalité de la Formation de Ghoundjaïa au niveau du Djebel M'daouer (Fig. 2 et Tab. 1) et chute ponctuellement à $80 \%$ juste après la limite cénomanienne-turonienne. A la base de la Formation de Ghoundjaïa dans la coupe de Chebket Tamednaïa (Fig. 6 et Tab. 1), les valeurs de calcimètrie oscillent entre $40 \%$ et $80 \%$, puis augmentent à proximité de la limite cénomanienne-turonienne pour chuter ensuite autour de $20 \%$ dans le Membre supérieur. On constate que ces faibles valeurs coïncident avec la chute de niveau marin.
Au niveau de Djebel M'daouer, la zone d'anomalie marquée par trois pics positifs du signal $\delta^{13} \mathrm{C}$ bien distincts (Fig. 2), est située entre les niveaux $\mathrm{Md}$ 39 et Md 55. Les valeurs s'accroissent au milieu du Membre inférieur de la Formation de Ghoundjaïa: Pic $1(\mathrm{Md} 40)=4,73 \%$. Le $\delta^{13} \mathrm{C}$ montre des valeurs élevées: Pic $2($ Md 50) $=5.43 \%$ au sommet de ce membre et atteint la valeur maximale enregistrée au niveau du pic $3(\mathrm{Md} \mathrm{55})=5,49 \%$ qui se trouve dans le début du Membre marneux médian (b) (Tab.1). Ensuite, la courbe isotopique chute vers des valeurs négatives le long du deux tiers restants de l'intervalle du Membre médian pour atteindre -1\% au niveau Md 61 et se stabilisent sur une grande partie du Membre supérieur, jusqu'à Md 68 (Tab. 1). Il faut noter que les échantillons de marnes Md 56, Md 57 , Md 58 et Md 59, correspondant à cet intervalle, n'ont pas subi d'analyse isotopique.

Le profil de la courbe d'analyse isotopique du $\delta^{13} \mathrm{C}$ dans la coupe de Tamednaïa présente moins nettement les trois anomalies positives (Fig. 6). La valeur du pic $1(\mathrm{TM} 19)=4,83 \%$ qui est maximale se trouve à la base du Membre calcaire inférieur de la Formation de Ghoundjaïa. Les deux autres valeurs des pic 2 (TM 27) et pic 3 (TM 32) $=2,75$ \%o sont égales et se trouvent au sommet du même membre (Tab.1). Après la surface bioturbée TM 33 
Table 1.- Valeurs des isotopes de $\delta{ }^{13} \mathrm{C}$ et de la calcimétrie des deux coupes étudiées de Djebel M'daouer et Chebket Tamednaïa.

M'daouer

\begin{tabular}{|c|c|c|}
\hline & Taux de & Moyenne \\
\hline échantillon & $\mathrm{CaCO}_{3}(\%)$ & $\delta^{13} \mathrm{C} / \mathrm{VPDB}$ \\
\hline Md-76 & 89.614 & -0.27 \\
\hline Md-74 & 95.655 & -0.69 \\
\hline Md-73 & 95.721 & -1.47 \\
\hline Md-72 & 94.365 & -2.16 \\
\hline Md-71 & 79.662 & -2.78 \\
\hline Md-70 & 86.421 & -3.85 \\
\hline Md-69 & 76.436 & -5.76 \\
\hline Md-68 & 93.043 & -1.37 \\
\hline Md-67 & 92.928 & -1.13 \\
\hline Md-66 & 87.975 & -3.05 \\
\hline Md-64 & 94.152 & -0.96 \\
\hline Md-63 & 92.624 & 0.43 \\
\hline Md-62 & 94.509 & -0.72 \\
\hline Md-61 & 94.979 & -1.01 \\
\hline Md-55 & 88.205 & 4.49 \\
\hline \multicolumn{3}{|c|}{ Limite Cénomanien-Turonien } \\
\hline Md-51 & 94.663 & 4.03 \\
\hline Md-50 & 91.700 & 5.43 \\
\hline Md-49 & 97.855 & 4.99 \\
\hline Md-48 & 92.428 & 5.06 \\
\hline Md-47 & 97.079 & 5.13 \\
\hline $\mathrm{Md}-46$ & 97.976 & 4.61 \\
\hline $\mathrm{Md} 45$ & 95.543 & 1.15 \\
\hline $\mathrm{Md} 44$ & 95.555 & 3.69 \\
\hline $\mathrm{Md}-43$ & 96.831 & 3.74 \\
\hline Md-42 & 92.925 & 3.75 \\
\hline $\mathrm{Md} 41$ & 94.102 & 4.00 \\
\hline $\mathrm{Md}-40$ & 95.103 & 4.73 \\
\hline Md 39 & 97.817 & 3.45 \\
\hline Md 38 & 89.233 & 2.95 \\
\hline Md 37 & 87.122 & 3.03 \\
\hline Md 35 & 91.725 & 1.13 \\
\hline Md 34 & 88.243 & 3.19 \\
\hline Md 32 & 91.226 & 3.23 \\
\hline Md 30 & 89.406 & 3.17 \\
\hline Md 29 & 87.978 & 2.29 \\
\hline \multicolumn{3}{|l|}{ Tamednaïa } \\
\hline TM-48 & 26.189 & -0.68 \\
\hline TM-47 & 22.104 & -2.00 \\
\hline TM-46 & 22.609 & -1.30 \\
\hline
\end{tabular}

Tabla 1.- Continued

\begin{tabular}{|c|c|c|}
\hline \multicolumn{3}{|l|}{ Tamednaïa } \\
\hline & Taux de & Moyenne \\
\hline échantillon & $\mathrm{CaCO}_{3}(\%)$ & $\delta^{13} \mathrm{C} / \mathrm{VPDB}$ \\
\hline TM-44 & 54.926 & -2.40 \\
\hline TM-43 & 97.083 & -8.20 \\
\hline TM-42 & 86.200 & -5.30 \\
\hline TM-39 & 91.623 & -2.55 \\
\hline TM-38 & 98.281 & -2.94 \\
\hline TM-36 & 95.714 & -4.37 \\
\hline \multicolumn{3}{|c|}{ Limite Cénomanien-Turonien } \\
\hline TM-32 & 68.926 & 2.75 \\
\hline TM-31 & 88.458 & 2.22 \\
\hline TM-27 & 38.201 & 2.75 \\
\hline TM-26 & 80.898 & -1.33 \\
\hline TM-24 & 9.515 & 4.63 \\
\hline TM-23 & 15.170 & 4.83 \\
\hline TM-22 & 15.685 & 4.43 \\
\hline TM-21 & 96.194 & 1.83 \\
\hline TM-20 & 66.698 & -0.11 \\
\hline TM-19 & 30.400 & 3.19 \\
\hline TM-18 & 75.403 & 0.75 \\
\hline TM-17 & 71.513 & 2.09 \\
\hline TM-16 & 91.155 & 1.67 \\
\hline
\end{tabular}

(discontinuité Sb) (Fig. 6, 7B), la courbe chute brutalement vers les valeurs isotopiques négatives pour atteindre un minimum de $-8.20 \%$ au niveau TM 43 au sein du Membre intermédiaire (Tab. 1). Cette valeur minimale peut être liée à la dolomitisation. La courbe isotopique reprend ensuite des valeurs positives le long du Membre supérieur.

A priori, la différence d'épaisseur de la Formation de Ghoundjaïa entre la coupe de Tamednaïa (44 m) et celle de M'daouer (92 m) suggère bien une différentiation paléobathymétrique offshore-foreshore attestée au point de vue sédimentologique (Fig. 9). Il est important de signaler l'anomalie positive du $\delta^{13} \mathrm{C}$ qui débute au milieu du Membre inférieur et se termine au début du Membre médian pour les deux coupes. Ces deux membres sont séparés par une discontinuité d'importance régionale délimitant ainsi deux grandes séquences SI et SII dont la première a une surface transgressive basale (ST) bien claire sous forme de discordance angulaire au niveau de Tamednaïa. La deuxième séquence de dépôt se termine par des cortèges de haut niveau aprés une 
surface d'inondation maximale (Sim) (Fig. 9). Dans la coupe de Tamednaïa (Fig. 6, 9), le Membre inférieur enregistre deux pics $1-2$ du $\delta^{13} \mathrm{C}$ qui coïncident avec deux phases de montée du niveau marin (Si1, $\mathrm{Si2}$ ) séparées par une brève chute. La troisième montée (Sim de la S3) qui passe au milieu du Membre médian, est inscrite hors de l'anomalie positive dans la zone de retrait du signal du $\delta^{13} \mathrm{C}$. Les fluctuations de la courbe $\delta^{13} \mathrm{C}$ dans la coupe de M'daouer (Fig. 2, 9) présente les trois pics distinctifs $(1,2$ et 3$)$ qui sont comparables aux ceux de la coupe de Tamednaïa (Fig. 9). Le premier pic coïncide aussi avec une montée marine (Si1). Les deux pics 1 et 2 se trouvent dans le Membre inférieur et le pic 3 se place déjà dans le Membre médian. La limite cénomanienne-turonienne passe juste avant le pic (3) et après la discontinuité régionale $(\mathrm{sf})$ de Djebel $\mathrm{M}$ 'daouer corrélable à celle de Tamednaïa.

A l'échelle régionale et mondiale, des exemples similaires de l'enregistrement de l'anomalie positive comme à la frontière algéro-tunisienne (Grosheny et al., 2013) et en Tunisie, où la Formation Bahloul comprend les trois pics I, II et III dont le premier débute dans le Pré-Bahloul (Barrett, 1998; Accarie et al., 1999; Accarie et al., 2000; Soua, 2006; Soua et Tribovillard, 2007, Soua et al., 2007) et la limite cénomanienne-turonienne se positionne après le retrait de l'anomalie positive, après le troisième pic. Les trois excursions positives ont été aussi observées au Tarfaya (Maroc) (Lebdel et al., 2013) et dans le Gabal Libni au nord de Sinai (Egypte) (El-Nady et Shahin, 2008).

Des excursions positives semblables ont été spectaculairement signalées dans le domaine des Apennins (Scholle et Arthur, 1980; Jenkyns et al., 1994; Erbacher et al., 1994), dans le SE de la France au sein du "Niveau Thomel" (Morel, 1998), et à Gubbio en Italie (Tsikos et al., 2004; Erbacher et al., 1996). Elles sont typiques et de courte durée, libellées "a", "b" et "c" à Eastbourne (Angleterre) (Pratt

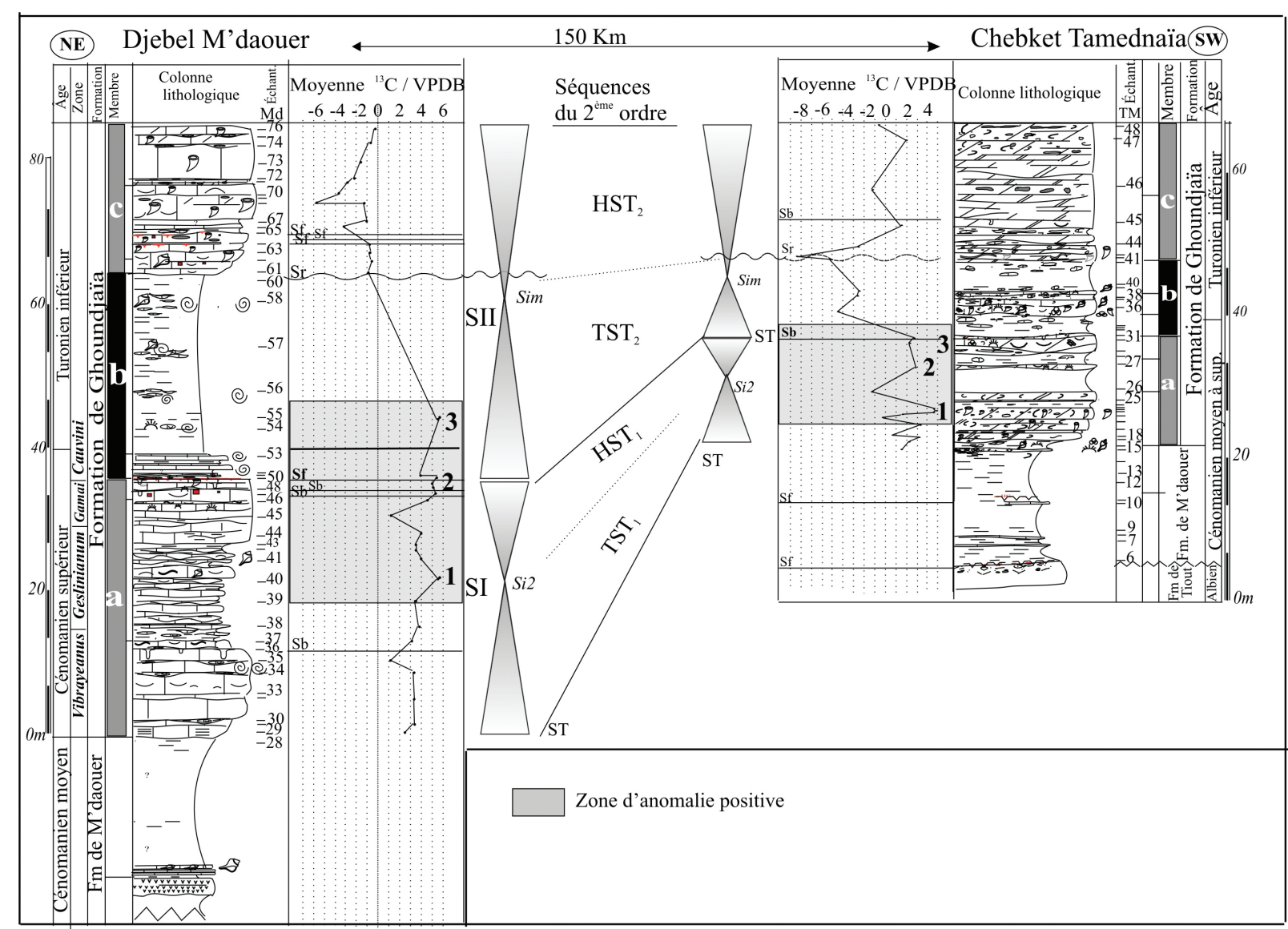

Fig. 9._- Corrélation entre les coupes de Djebel M'daouer et de Chebket Tamednaïa. Voir légendes sur la figure 6. 
et Threkeld, 1984; Gale et al., 2005; Jarvis et al., 1988), et dans le Colorado, dans la coupe de Pueblo (Leckie, 1985; Keller et al., 2004).

\section{Discussion}

Bassoullet (1973) cite un niveau à Vascoceras cf. gamai (Choffat, 1897) dans le banc ferrugineux qui termine la première corniche calcaire (B1), correspondant au Membre carbonaté inférieur (a). Boualem (2006) a également récolté Vascoceras gamai (Choffat, 1897) (détermination par Atrops) dans ce même banc scellé par une discontinuité très nette qui a une large extension matérialisée par un surface durcie. Sur la coupe du Djebel M'daouer, il est donc intéressant de noter la présence de Vascoceras gamai (Choffat, 1897) sous le niveau Vascoceras cauvini (Chudeau, 1909), ce qui recoupe les observations faites par Amard et al. (1981), Luger et Gröschke (1989); Meister et Piuz (2013). Les deux biozones d'ammonites Neolobites Vibrayeanus (d'Orbigny, 1841) et Vascoceras gamai (Choffat, 1897) enregistrées dans la Formation de Ghoundjaïa de cette coupe, ont permis de situer avec imprécision la limite cénomanienne-turonienne qui était tracée d'une façon imprécise par les prédécesseurs.

Dans le Turonien inférieur, les ammonites sont toujours rares à travers les Monts des Ksour. Seule la présence, dans la partie supérieure du Membre marneux médian, de Leoniceras (Bassoulet, 1973), genre cité en Espagne dans la sous-zone à Luciae (Barroso-Barcenilla et Goy, 2007), de Mammites sp. et Thomasites sp. (Cornet, 1952), indique le Turonien inférieur. Des Vascoceras fragmentaires trouvés dans le Membre médian de la coupe de M'daouer, au-dessus du niveau riche en Vascoceras cauvini (Chudeau, 1909), sont, difficiles à déterminer spécifiquement. Un spécimen mieux conservé, provenant de la partie supérieure du Membre marneux (banc Md 58) est attribué à Vascoceras cf. cauvini (Chudeau, 1909) (Fig. 3F). Cette espèce pourrait donc encore persister à la base du Turonien, en association avec des formes du Turonien inférieur.

Au sein de la Formation de Ghoundajaïa, les organismes planctoniques ont enregistré des changements importants dans la composition du biofaciès (Fig. 2, 6). Ainsi, l'assemblage de faunes, relativement diversifié, observé dans le Membre inférieur, est dominé par des Hedbergella sp. (Fig. 4H, Md 38) et Whiteinella sp. à la base, associée à des Neolobites (Fig. 3C); tandis qu'au sommet de ce membre, les Heterohelix sp. (Md 52, Fig. 5C) sont accompagnés de Vascoceras cf. cauvini (Chudeau, 1909) (Fig. 3F, Md 58,) qui persistent jusqu'au Membre médian. On note une disparition progressive des foraminifères vers le haut. Ce bioévènement s'avère universel, comme il a été signalé dans la plupart des séries cénomanienne-turoniennes à faciès océanique. Il coïncide aussi avec le "Shift Heterohelix" (O'Dogherty, 1994; O’Dogherty et Guex,2001; Soua, 2005; Soua et Zaghbib-Turki, 2006; Ruault-Djerrab et al., 2012). La microfaune disparaît au Turonien dans les deux coupes étudiées de M'daouer et de Tamednaïa.

Dans les Monts des Ksour, l'événement anoxique océanique du Cénomanien-Turonien n'a pas donné de "black-shales". Dans le Membre inférieur de la Formation de Ghoundjaïa de M'daouer, on enregistre une absence momentanée des foraminifères et ammonites (Fig. 2), qui coïncide avec le début de l'anomalie positive $\delta^{13} \mathrm{C}$ (pic 1) et la deuxième inondation (Si2). Elle est suivie par un renouvellement faunique durant le deuxième cycle transgressif (autour du pic 2 et 3). On peut expliquer cette raréfaction ou absence de la faune comme due au retrait d'organismes suite à l'installation de conditions environnementales défavorables probablement en liaison avec la diminution d'oxygène (l'anoxie) lors de la phase transgressive du Cénomanien supérieur (Schlanger \& Jenkys, 2007). Comme on peut considérer cette raréfaction de la faune comme équivalente des niveaux profonds à "black-shales" qui sont bien enregistrés dans les Aurès de l'est algérien (Grosheny et al., 2013), ceci est probablement lié à l'incidence de la stagnation des eaux sur cette partie de plate-forme carbonaté en formant ainsi une zone à oxygène minimum qui n'atteignait pas les eaux de surface où vivaient les foraminifères globuleux non carénés, comme les Whiteinella et Heterohelix.

Dans la coupe de M'daouer, seuls les deux premiers pics d'anomalies isotopiques $\delta^{13} \mathrm{C}$ ont été enregistrés; le pic 1 au milieu de la zone à Geslinianum du Cénomanien supérieur et le pic 2 dans la zone Gamai proche de la limite cénomanienne-turonienne (Fig. 2). La montée de la courbe au début du pic 1 dans la zone Vibrayeanus de la coupe de M'daouer est équivalente à la zone Calycoceras (Proeucalycoceras) 
guerangeri (Spath, 1926) de l'Europe. Le pic 2 de la même coupe, très proche, au-dessous de la limite cénomanienne-turonienne est équivalent au pic "c" en référence à la coupe d'Eastbourne (UK), où cette limite se place juste au-dessus (Paul et al.,1999; Jarvis et al., 2006; Voigt et al., 2008). Le troisième pic de la coupe de M'daouer se trouve déjà dans la zone Cauvini du Turonien inférieur. Au-dessus de ce dernier, la courbe isotopique chute brusquement de 5.49 $\%$ à $-1 \%$, probablement en rapport avec une lacune entre les membres médian et supérieur. D'ailleurs, la barre carbonatée supérieure du Membre supérieur superpose aux marnes du Membre médian avec une surface ravinante $(\mathrm{Sr})$ bien marquée en affleurement (Fig. 2 et Fig. 3A). Cette discontinuité peut être tracée régionalement et peut être comparée à l'érosion de sub-Plenus d'Eastbourne (UK) (Paul et al., 1999) et au changement du faciès de la base de la coupe de Thomel au pont d'Issole (Danzelle, 2019).

\section{Conclusion}

Les données paléontologiques ont permis d'individualiser les zones d'ammonites: Virayeanus, Gamai et Cauvini dans la coupe de M'daouer des Monts des Ksour de l'Atlas saharien. Les données isotopiques de l'excursion positive du $\delta{ }^{13} \mathrm{C}$ fournies par les coupes de M'daouer et Tamednaïa couplées aux données de la biostratigraphie ont, d'une part, facilité le traçage de la limite cénomanienne-turonienne, et d'autre part, de corréler localement la Formation de Ghoundjaïa dans les deux coupes étudiées. Le signal $\delta^{13} \mathrm{C}$ de la zone d'anomalie positive a fait ressortir les trois pics caractéristiques du passage cénomanienne-turonienne et qui sont utilisés pour une corrélation locale avec la coupe de Tamednaïa dépourvue de faune biostratigraphique valable. La configuration du signal $\delta^{13} \mathrm{C}$ avec ses trois pics dans la zone d'anomalie positive peut être comparé avec d'autres coupes à l'échelle régionale et même globale. Dans cette série de plate-forme étudiée des Monts des Ksour, les fluctuations de la courbe $\delta^{13} \mathrm{C}$ associeés à l'analyse séquentielle ont permis de mettre en évidence des montées de niveau marin au cours du Cénomanien supérieur et une inondation maximale en coïncidence avec la limite cénomanienne-turonienne.

\section{REMERCIEMENTS}

Nos remerciements s'adressent F. Martinot et F. Fourel qui ont réalisé au laboratoire de Géologie de l'université de Lyon I les analyses géochimiques ainsi que de nombreuses lames minces. Également, nous remercions C. Lecuyer qui a autorisé à faire ces analyses. J'adresse aussi les remerciements aux membres du Centre de Recherche et Développement de Boumerdès qui ont confectionné une partie de lames minces. Nous remercions infiniment le rédacteur en Chef J.M. Cebriá pour la prise en charge de la présente note. Nous remercions beaucoup les deux reviewers pour leurs commentaires minutieux sur le contenu du manuscrit et leur contribution à l'amélioration de sa qualité scientifique. Enfin, sans oublier toutes les autorités civiles et militaires qui m'ont facilité l'accès au terrain dans des moments difficiles.

\section{Références}

Accarie, H.; Emmanuel, L.; Robaszynski, F.; Baudin, F.; Amedro, F.; Caron, M. \& Deconinck, J.-F. (1996). La géochimie isotopique du carbone ( $\delta 13 C)$ comme outil stratigraphique. Application à la limite Cénomanien-Turonien en Tunisie centrale. Compte Rendu de l'Academie des Sciences, Paris, 322(IIa): 579-586.

Accarie, H.; Robaszynski, F.; Amedro, F.; Caron M. \& Zagrani, M.F. (2000). Stratigraphie événementielle au passage Cénomanien-Turonien dans le secteur occidental de la plate-forme de Tunisie centrale (Formation Bahloul, région Kalaat Senan). Annales des mines et de la Géologie, Tunisie, 40: 63-80.

Amard, B.; Collignon, M. \& Roman, J. (1981). Etude stratigraphique et paléontologique du Crétacé supérieur et Paléocène du Tinrhert-West et Tademaït-Est (Sahara algérien). Documents des laboratoires de géologie de Lyon, H.S. 6: 15-173.

Amedro, F.; Busson, G. \& Cornet, A. (1996). Révision des ammonites du Cénomanien supérieur et du Turonien inférieur du Tinrhet (Sahara algérien): implications biostratigraphiques. Bulletin du Muséum National d'Histoire Naturelle. 4ème série, section C, Sciences de la Terre, Paléontologie, Géologie, Minéralogie, Paris, 18: 179-232.

Aris, Y.; Coiffait, P. E. \& Guiraud, M. (1998). Characterization of Mesozoic-Cenozoic deformations and paleostress field in the central Constantinois, northeast Algeria. Tectonophysics, 290: 59-85. https://doi. org/10.1016/S0040-1951(98)00012-2

Arthur, M.A.; Dean, W. E. \& Pratt, M. (1988). Geochemical and climatic effects of increased marine organic carbon burial at the Cenomanian-Turonian boundary. Nature, 335: 714-717. https://doi. org/10.1038/335714a0 
Barrett, P. (1998). A comparative organic geochemical and stable isotope study of the Cenomanian-Turonian organic rich-sediment from Tunisia, Germany and the UK. PhD thesis, University of New Castle, $250 \mathrm{pp}$.

Bassoullet, J.P. (1973). Contribution à l'étude stratigraphique du Mésozoïque de l'Atlas saharien occidental (Algérie). PhD thesis, Université de Paris, $497 \mathrm{pp}$.

Boualem, N. (2006). Dynamique sédimentaire du Crétacé des Djebels Rhoundjaia et M'Daouer (région d'Aïn Ouarka, Monts des Ksour, Algérie). Mémoire de Magister en Sciences de la Terre de 1'Université d'Oran, $127 \mathrm{pp}$.

Busson, G.; Dhondt, A.; Amédro, F. \& Néraudeau, D. (1999). La grande transgression du Cénomanien supérieur-Turonien inférieur sur la Hamada de Tinrhert (Sahara algérien): datations biostratigraphiques, environnement de dépôt et comparaison d'un témoin épicratonique avec les séries contemporaines à matière organique du Maghreb. Cretaceous research, 20 (1): 29-46. https://doi.org/10.1006/cres.1998.0137

Collignon, M. (1957). Céphalopodes néocrétacés du Tinrhert (Fezzan). Annales de Paléontologie, 43: 113-136.

Collignon, M. (1965). Nouvelles ammonites néocrétacées sahariennes. Annales de Paléontologie (Invertébrés), 5: $165-202$

Conrad, G. (1969). L'évolution continentale post-hercynienne du Sahara algérien: (Saoura, Erg Chech-Tanezrouft, Ahnet-Mouydir). Editions du Centre National des Recherches Scientifiques, 527 pp.

Cornet, A. (1952). L'Atlas saharien sud-oranais. XIX Congrès Géologique International, Alger, 51 pp.

Danzelle, J. (2019). Caractérisation géochimique des changements paléoenvironnementaux au cours de l'Évènement Océanique Anoxique 2 ( 94 Ma) dans le Bassin Vocontien (SE France). PhD thesis, Institut des Sciences de la Terre, Paris, 292 pp.

Dunham, R. J. (1962). Classification of carbonate rocks according to depositional texture. (Ham, W. E. Eds.), Classification of carbonate rocks. American Association of Petroleum Geologists Memoir, 108-121.

El-Nady, H. \& Shahin, A. (2008). Biostratigraphy and stable isotopic ( $\delta 13 \mathrm{C}$ and $\delta 18 \mathrm{O}$ ) record across the Cenomanian-Turonian boundary in Gabal Libni section, northern Sinai, Egypt: an integrated study. 10th International Conference on the Geology of the Arab World (Gaw10), Giza, Egypt, 36 pp.

Erbacher, J. (1994). Entwicklung und Palaoozeanographie mittelkretazischer Radiolarien der westlichen Tethys (Italien) und des Nordatlantiks. PhD thesis, Eberhard-Karls-Universitat, 119 pp.

Erbacher,J.; Thurow J.\&Littke, R.(1996). Evolution patterns of radiolaria organic matter variations: a new approach to identify sea-level changes in mid-Cretaceous pelagic environments. Geology, 24(6): 499-502. https://doi.
org/10.1130/0091-7613(1996)024<0499:EPORAO> 2.3. $\mathrm{CO} ; 2$

Flamand, G.B.M. (1911). Recherches géologiques et géographiques sur le Haut Pays de l'Oranie et sur le Sahara. PhD thesis, Faculté des Sciences de l'Université de Lyon, Lyon, 1001 pp.

Folk, R.L. (1959). Practical petrographic classification of limestones. American Association of Petroleum Geologists Bulletin, 43: 1-38. https:// doi.org/10.1306/0BDA5C36-16BD-11D7$8645000102 \mathrm{C} 1865 \mathrm{D}$

Frizon De Lamotte, D.; Saint Bezar, B.; Bracene, R. \& Mercier, T. (2000). The two main steps of the Atlas building and geodynamics of the West Mediterranean. Tectonics, 19(4): 740-761. https://doi. org/10.1029/2000TC900003

Gale, A.S.; Kennedy, W.J.; Voigt, S. \& Walaszczyket, I. (2005). Stratigraphy of the Upper Cenomanian-Lower Turonian Chalk succession at Eastbourne, Sussex, UK: ammonites, inoceramid bivalves and stable carbon isotopes. Cretaceous Research, 26(3): 460-487. https://doi.org/10.1016/j.cretres.2005.01.006

Guiraud, R. (1990). Evolution post-triasique de l'avantpays de la chaine alpine en Algérie. Office national de la géologie, Mémoire, 3: 266 pp.

Grosheny, D.; Ferry S.; Jati, M.; Ouaja, M.; Bensalah, M.; Atrops, F.; Chikhi-Aouimer, F.; BenkheroufKechid, F.; Hedi, N. \& Ait-Salem, H. (2013). The Cenomanian-Turonian boundary on the Saharan Platform (Tunisia and Algeria). Cretaceous Research, 42: 66-84. https://doi.org/10.1016/j. cretres.2013.01.004

Herkat, M. \& Delfaud, J. (2000). Genèse des séquences du Crétacé supérieur des Aurès (Algérie). Rôle de l'eustatisme, de la tectonique et de la subsidence: une mise au point. Compte Rendu de l'Accadémie des Sciences, série IIa, 330: 785-792. https://doi. org/10.1016/S1251-8050(00)00229-9

Herkat, M. (2001). Eustatic and paleogeographic control on the Aurès basin Upper Cretaceous sedimentation (Algeria). 21th International Association sedimentologic meeting, Davos, 72.

Jarvis,I.; Carson, G.; Cooper,M.; Hart, M.;Leary,P.; Tocher, B.; Horne, D. \& Rosenfield, A. (1988). Microfossil assemblages and the Cenomanian-Turonian (Late Cretaceous) OAE. Cretaceous Research, 9: 3-103. https://doi.org/10.1016/0195-6671(88)90003-1

Jarvis, I.; Gale, A.S.; Jenkyns, H.C. \& Pearce, M.A. (2006). Secular variation in Late Cretaceous carbon isotopes and sea-level change: evidence from a new $\delta 13 \mathrm{C}$ carbonate reference curve for the Cenomanian-Campanian (99.6-70.6 Ma). Geologic Magazine, 143: 561-608. https://doi.org/10.1017/ S0016756806002421

Jenkyns, H.C.; Gale, A. S. \& Corfield, R. M. (1994). Carbon and oxygen isotope stratigraphy of the English Chalk 
and Italian Scaglia and its palaeoclimatic significance. Geological Magazine, Cambridge University, 131(1): 1-34. https://doi.org/10.1017/S0016756800010451

Kazi-Tani, N. (1986). Evolution géodynamique de la bordure nord-africaine: le domaine intraplaque nord algérien, approche séquentielle. $\mathrm{PhD}$ thesis, Université de Pau et des Pays de l'Adour, Pau, 871 pp.

Keller, G.; Stuben, D.; Zsolt, B. \& Adatte, T. (2004). Cenomanian-Turonian sea level and salinity variations at Pueblo, Colorado. Palaeogeography, Palaeoclimatology, Palaeoecology, 211: 19-43. https://doi. org/10.1016/j.palaeo.2004.04.003

Laville, E. (1985). Evolution sédimentaire, tectonique et magmatique du bassin jurassique du Haut-Atlas (Maroc): Modèle en relais multiple décrochement. $\mathrm{PhD}$ thesis, Université de Montpelier, Montpelier, $166 \mathrm{pp}$.

Lebdel, V.; Lezin, C.; Andreu, B.; Wallez M.-J.; Ettachfini, El.-M. \& Riquier, L. (2013). Geochemical and palaeoecological record of the CenomanianTuronian Anoxic Event in the carbonate platform of the Preafrican Trough, Morocco. Paleogeography, Paleoclimatology Paleoecology, 369: 79-98. https:// doi.org/10.1016/j.palaeo.2012.10.005

Leckie, R.M. (1985). Foraminifera of the CenomanianTuronian boundary interval, Greenhorn Formation, Rock Canyon Anticline, Pueblo, Colorado. In: FineGrained Deposits and Biofacies of the Cretaceous Western Interior Seaway: Evidence of Cyclic Sedimentary Processes (Pratt, L.M.; Kauffman, E.G. \& Zelt, F.B., Eds.), Field Trip Guidebook, 4: 139-149. https://doi.org/10.2110/sepmfg.04.139

Luger, P. \& Gröschke, M. (1989). Late Cretaceous ammonites from the Wadi Gena area in the Egyptian Eastern. Palaeontology, 32(2): 355-407.

Meister, C. \& Puiz, A. (2013). Late Cenomanian-Early Turonian ammonites of the Southern Tethys margin from Morocco to Oman: biostratigraphy, paleogeography morphology. Cretaceous Research, 44: 83-103. https://doi.org/10.1016/j.cretres.2013.03.009

Morel, L. (1998). Stratigraphie à haute résolution du passage Cénomanien-Turonien. PhD thesis, Université Pierre et Marie Curie, Paris, 236 pp.

O’Dogherty, L. (1994). Biochronology and paleoecology of Mid-Cretaceous radiolarians from Northern Apennines (Italy) and Betic Cordillera (Spain). Mémoires de Géologie de l’Université de Laussane, 21: 1-415.

O’Dogherty, L. \& Guex, J. (2001). Rates and Pattern of Evolution among Cretaceous Radiolarians: Relations with Global Paleoceanographic Events. Micropaleontology, 48(1): 1-22.

Paul, C.R.C.; Lamolda, M.A.; Mitchell, S.F.; Vaziri, M.R.; Gorostidi, A. \& Marshall, J.D. (1999). The Cenomanian-Turonian boundary at Eastbourne (Sussex, UK): a proposed European reference section. Palaeogeographic Palaeoclimatologic Palaeoecologic,
150(1-2): $\quad 83-121 . \quad$ https://doi.org/10.1016/ S0031-0182(99)00009-7

Pratt, L.M. \& Threkeld, C.N., (1984). Stratigraphy significance of $13 \mathrm{C} / 12 \mathrm{C}$ Ratios in Mid-Cretaceous Rocks of the Western Interior, U.S.A. In: Mesozoic of Middle North America: A Selection of Papers from the Symposium on the Mesozoic of Middle North America (Stott, D.F. \& Glass, D.J., Eds.), Canadian Society of Petroleum Geologists Memoir, 9: 305-312.

Ruault-Djerrab, M.; Ferre, B. \& Kechid-Benkherouf, F. (2012). Etude micropaléontologique du CénomanoTuronien dans la région de Tébessa (NE Algérie): implications paléoenvironnementales et recherche de l'empreinte de l'OAE2. Revue de Paléobiologie, 31 (1): 127-144.

Schlanger, S.O. \& Jenkyns, H.C. (1976). Cretaceous Oceanic Anoxic Events: Causes and consequences. Géologie en Mijenbouw, 55 (3-4): 179-194.

Schlanger, S.O.; Arthur, M.A.; Jenkyns, H.C. \& Scholle, P.A. (1987). The Cenomanian-Turonian oceanic anoxic event: I. Stratigraphy and distribution of organic-rich beds and the marine 13C excursion. Geological Society Special Publication, 26: 371-399. https://doi.org/10.1144/GSL. SP.1987.026.01.24

Schlanger, S.O. \& Jenkyns, H.C. (2007). Cretaceous oceanic anoxic events: causes and consequences. Netherlands Journal of Geosciences, Geology, 55 (34): 179-184.

Scholle, P. A. \& Arthur M. A. (1980). Carbon isotope fluctuation in cretaceous pelagic limestones: potential stratigraphic and petroleum exploration tool. American Association of Petroleum Geology Bulletin, 64 (1): 67-87.

Soua, M. (2005). Biostratigraphie de haute résolution des foraminifères planctoniques du passage Cénomanien-Turonien et impact de l'événement anoxique EAO-2 sur ce groupe dans la marge sud de la Téthys, exemple régions de Jerissa et Bargou. MsC thesis, Université de Tunis, El Manar, 169 pp.

Soua, M., (2006). High-resolution biotic records of the Bahloul Formation (OAE-2, Latest Cenomanianearly Turonian) from Jerissa and Bargou areas, Northwestern and North-central Tunisia. Proceedings of the Exploration and Production Conference, New Orleans, 10: 177-185.

Soua, M. \& Zaghbib-Turki D. (2006). Cenomanian-Turonian Radiolarian assemblages at Oued El Kharroub section (North-Central Tunisia). Association Tunisienne des Etudes Internationales de Géologie, 2: 67-78.

Soua, M. \& Tribovillard, N. (2007). Depositional model at the Cenomanian-Turonian boundary for the Bahloul Formation, Tunisia. Comptes Rendus Geoscience, 339 (10): 692-701. https://doi. org/10.1016/j.crte.2007.08.002 
Soua, M.; Chebbi, M.R. \& Gharsalli R. (2007). Highresolution sequence stratigraphic and cyclostratigraphic interpretation of a basal transgressive system tract in North-central Tunisia: the Cenomanian-Turonian Bahloul Formation. Geophysical Research Abstracts, 9: 3.

Tsikos, H.; Jenkyns, H.C.; Walsworth-Bell, B.; Petrizzo, M.R.; Forster, A.; Kolonik, S.; Erba Premoli, E.; Silva, I.; Baas, M.; Wagner, T. \& SinninghDamsté, J.S. (2004). Carbon-isotope stratigraphy recorded by the Cenomanian-Turonian Oceanic
Anoxic Event: correlation and implications based on three key localities. Journal of the Geological Society, 161 (4): 711-719. https://doi. org/10.1144/0016-764903-077

Voigt, S.; Erbacher, J.; Mutterlose, J.; Weiss, W.; Westerhold, T.; Wiese, F.; Wilmsen, M. \& Wonik, T. (2008). The Cenomanian-Turonian of the Wunstorf section (North Germany): global stratigraphic reference section and new orbital time scale for oceanic anoxic event 2. Newsletters on Stratigraphy, 43: 65-89. https://doi.org/10.1127/0078-0421/2008/0043-0065 